\title{
PRINCIPALES INSECTOS PERFORADORES DE FRUTOS EN ALGUNOS FRUTALES DE LA ZONA DE SATIPO*
}

\author{
Alomía Lucero, José Manuel ${ }^{1}$ \\ Facultad de Ciencias Agrarias - Satipo. Universidad Nacional del Centro del Perú
}

\section{RESUMEN}

La investigación se desarrolló considerando la importancia que tienen los insectos perforadores de frutos y las grandes pérdidas que se tienen en el guanábano Anona muricata, Caimito chrisophyllum y zapote Matisia cordata en la zona de Satipo. El trabajo consistió en la colección mensual de frutos y el conteo de larvas por fruto durante todo el año, para ello la zona de muestreo tuvo a tres lugares de colección, tomando como muestra representativa 5 plantas de guanábano, 3 plantas de caimito y 2 plantas de zapote por cada lugar ; asimismo, se realizó la recuperación de adultos e identificación por comparación y claves taxonómicas; los datos estadísticos fueron procesados en cuadros de porcentaje y gráficos de histogramas. Los resultados fueron los siguientes: en los frutos de guanábano se ha encontrado a Cerconata anonnella Sepp., un Lepidóptero - Oecophoridae, al estado larval barrenador, considerado plaga principal cuya infestación alcanza el $100 \%$ durante todo el año, aunque con daños parciales en la pulpa, su población disminuye relativamente entre julio y noviembre, también se ha reportado a la avispa barrenadora de frutos Bephrata macullicolis; en el caimito se ha detectado la presencia de un díptero, mosca amarilla no identificada que no pertenece a la familia de los Teprhitidae, la infestación es del 96,5\% y se presenta sólo en los meses de noviembre a marzo, alcanzando la mayor población entre enero y febrero, esta plaga comparte espacio con la Anastrepha leptozona Hendel que se encuentra en menor proporción; y en el zapote se ha detectado a la mosca de la fruta Anastrepha kuhlmanni Lima como plaga principal, cuya infestación alcanza 37,02\% y se presenta en los meses de enero a marzo, asimismo se ha detectado la presencia de un lepidóptero barrenador que destruye la semilla que alcanza un $23,64 \%$ de infestación.

Palabras clave: guanábano, caimito, zapote, cerconata, anastrepha.

\section{MAIN FRUIT PERFORATOR INSECTS IN SOME FRUITS IN SATIPO AREA}

\begin{abstract}
The present investigation work was developed considering the importance the perforating insects have in the fruit and the great loses that cause in the guanabana (Anona muricata) caimito (Caimito chrisophyllum) and zapote (Matisia cordata) in the area of Satipo. This research consisted in the monthly collection of fuits and the count of la rvas per fruit through all the year. Frothis purpose the gone had 3 areas of collection takingas representative sample 5 guanabana plants, 3 coimito plants and 2 zapote plants inyeach place; at the same time, also it was carried out the recoyection of the adults, and identifying by comparison taxonomic feaures. The autentic informations were procemedin $\%$ values aud histograms; the results were the following: in the ousobono Cerconata anonne sepp, a lepidoptero, oechoforidoc, were found at the lord state of barrenedor, considered the many plogne which affection reaches to $100 \%$, all the year long although with partiad damages in the pulp its population diminiches relatively fron July to November. Its alsohsne reported the baborer wasp of fruis. Bephrata macullicolis. In the coimito, its been detected the presence of a dipterus, mosca amarilla non identified that docent belong to the tephortidose family the infectation is arond $96.5 \%$ and its present only in the months of Novemberer to March, reaching its highest population between January and February this plague shyows presence with Anastrepha leptozona Heudel in a small quantity and in zopote its been detected the mosea of frui't Anastrepha kuhlmanni Lima as a main plague. Where infestation $37.02 \%$ in the months between January and March. Its also been detected the presence of a lepidopters bloser that destroys the seeds reaching a $23,6 \mathrm{y} \%$ of infestation
\end{abstract}

Key words: Guanabana, caimito, zapote, cerconata, anastrepha.

* Este trabajo de investigación fue recibido el 20/04/2007 retornado para su revisión 20/06/2007 y aprobado para su publicación 10/12/2007

1. E-mail: manuelalomia38@hotmail.com 


\section{INTRODUCCIÓN}

La zona de Satipo, es una importante región frutícola, siendo los cítricos uno de los principales cultivos, en el cual la mosca Anastrepha fraterculus Wied. es la plaga principal pero aparte de los cítricos contamos con una diversidad de especies de frutales nativos, que son un recurso potencial, pero que cada año se pierden en gran cantidad por los daños que ocasionan los insectos barrenadores de frutos, los mismos que al no ser estudiados no es posible emprender medidas de control efectivas.

Los órganos infestados en sus primeros estados suelen caerse prematuramente; muchos frutos atacados tardíamente se pudren y caen, otros se momifican y deshidratan en la planta; asimismo, la destrucción de los frutos suele tener gran importancia económica, ya que por lo general, ellos constituyen el propósito del cultivo (Cisneros V.y Fausto H., 1995).

En el guanábano se tienen plagas importantes a nivel de fruto, como la broca del fruto Cerconata anonella, cuyas larvas afectan frutos jóvenes causando grandes y profundas heridas; y la avispa del guanábano: Bephrata macullicolis, cuya larva vive en las semillas y luego pasa a la pulpa al madurar la fruta (PROEXANT, 2000; Minag, 2001; Mag, 1991; Sibaja, Ch. y Sanabria, U., 2002).

Para la investigación se tuvo como material vegetal a plantas de guanábano, zapote y caimito, por ser los frutales que alcanzan los mayores daños de perforadores de frutos, y a la vez, constituyen un recurso potencial cuyas frutas alcanzan buenos precios en los mercados locales, regionales y nacionales; pero se pierden gran parte de ellos por insectos perforadores, que no simplemente se trata de moscas de la fruta del género Anastrepha, sino de otros insectos; los mismos que requieren ser estudiados para emprender un control adecuado y fomentar su aprovechamiento y cultivo en los agricultores.

La identificación es importante, dado que se piensa que los frutales nativos son hospederos de la mosca de la fruta que afecta a los cítricos, mangos y otros. Las especies de Anastrepha son específicos con su planta frutal, a excepción de Anastrepha fraterculus que tiene una gama de hospederos (Pariona, V. 2004). Asimismo, la infestación es importante para determinar pérdidas económicas, y la fluctuación poblacional nos permite conocer el momento para aplicar medidas de control adecuadas.

La hipótesis de la investigación fue: "Las principales plagas de los frutales de la zona de Satipo, son principalmente larvas de lepidópteros y dípteros que causan serios daños durante los meses de producción"

Los objetivos de investigación fueron:
- Determinar las principales especies de insectos barrenadores de frutos en la zona de Satipo.

- Determinar la infestación que causan las principales especies de insectos barrenadores de frutos en la zona de Satipo.

- Determinar la fluctuación poblacional durante el año, de los principales insectos barrenadores de frutos en la zona de Satipo.

\section{MATERIAL Y MÉTODOS}

El trabajo fue desarrollado en la zona de Satipo, región Junín, en tres lugares de muestreo (Timarini, Satipo y Río Negro), cuya ubicación geográfica está a $660 \mathrm{msnm}$, con una temperatura media de $26^{\circ} \mathrm{C}$ y humedad relativa media de $85 \%$. Se trabajó con plantaciones frutales en producción durante un año, desde marzo del 2006 a marzo del 2007.

Se utilizaron frascos de recuperación de insectos, ligas, etiquetas, alfileres, tecnopor, tul, caja entomológica. También se utilizó un estereoscopio y una cámara digital.

El diseño de investigación fue no experimental, descriptivo. La población estuvo dada por los frutales de la zona de Satipo como el guanábano, caimito y zapote. Para la muestra se tomó al azar 5 frutales de guanábano, 3 de caimito y 2 de zapote en tres lugares de Satipo. Las variables independientes fueron especie de plaga y especie frutal; las variables dependientes fueron: número de frutos afectados por planta y número de larvas por fruto. Se hizo la recolección mensual de frutos y la recuperación de adultos para la identificación. Los insectos recolectados fueron identificados por comparación con claves taxonómicas. Los datos fueron procesados en cuadros de porcentaje, tablas y gráficos.

\section{RESULTADOS}

En el cuadro 1, se muestra que la polilla del fruto Cerconata anonella (Foto 1) infesta durante todo el año y a todos los frutos de guanábano (100\%), debido a que esta planta produce continuamente alimento para que la plaga haga varios ciclos al año. Pero se debe resaltar, que el daño directo realiza sólo a una parte del fruto, por lo que el resto de la pulpa puede ser aprovechada, aunque los frutos con daño no son posibles de comercializar por la consecuente pudrición secundaria.

Cuadro 1.- Insectos barrenadores registrados en el guanábano Anona muricata en la zona de Satipo.

\begin{tabular}{|c|c|c|c|}
\hline $\mathrm{N}^{0}$ & Orden & Familia & Especie \\
\hline 1 & Lepidoptera & Oecophoridae & $\begin{array}{l}\text { Cerconata } \\
\text { anonnella Sepp. }\end{array}$ \\
\hline 2 & Diptera & $\mathrm{nn}$ & $\mathrm{nn}$ \\
\hline
\end{tabular}




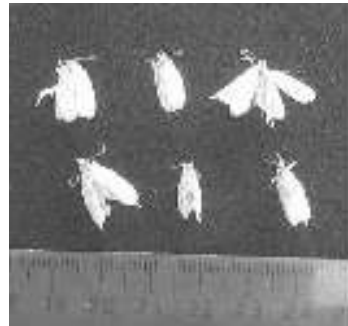

Foto 1. Fotografía digital de Cerconata anonella recuperados en la zona de Satipo.

Cuadro 2. Insectos barrenadores barrenadores registrados en el caimito en la zona de Aclipo.

\begin{tabular}{ccll}
\hline $\mathrm{N}^{0}$ & Orden & Familia & Especie \\
\hline 1 & Diptera & $\mathrm{nn}$ & $\mathrm{nn}$ \\
2 & Diptera & Tephritidae & $\begin{array}{l}\text { Anastrepha } \\
\text { leptozona } \\
\text { Hendel }\end{array}$ \\
\hline
\end{tabular}

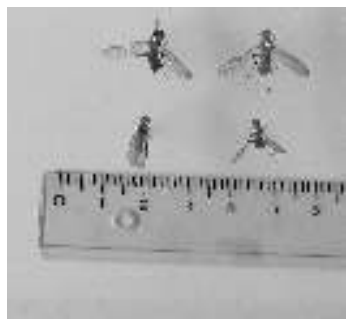

Foto 2.- Fotografía digital de la mosca amarilla del caimito (Díptero no identificado).

Cuadro 3.- Insectos barrenadores registrados en el zapote Matissia cordata en la zona Satipo.

\begin{tabular}{llll}
\hline $\mathrm{N}^{0}$ & Orden & Familia & Especie \\
\hline 1 & Diptera & Tephritidae & Anastrepha kuhlmanni Lima \\
2 & Lepidoptera & $\mathrm{nn}$ & $\mathrm{nn}$ \\
\hline
\end{tabular}

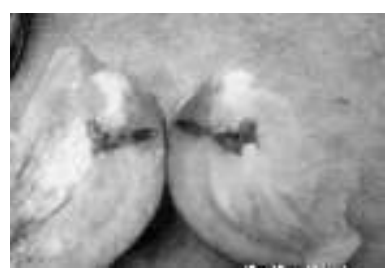

Foto 3.- Daños de la polilla barrenadora del zapote.

Fuente: José Alomía Lucero. (Satipo, febrero 2007)

En el gráfico 1, se muestra que la polilla del fruto Cerconata anonella infesta durante todo el año y a todos los frutos de guanábano (100\%), debido a que esta planta produce continuamente alimento para que la plaga haga varios ciclos al año. Pero se debe resaltar, que el daño directo realiza sólo a una parte del fruto, por lo que el resto de la pulpa puede ser aprovechada, aunque los frutos con daño no son posibles de comercializar por la consecuente pudrición secundaria.

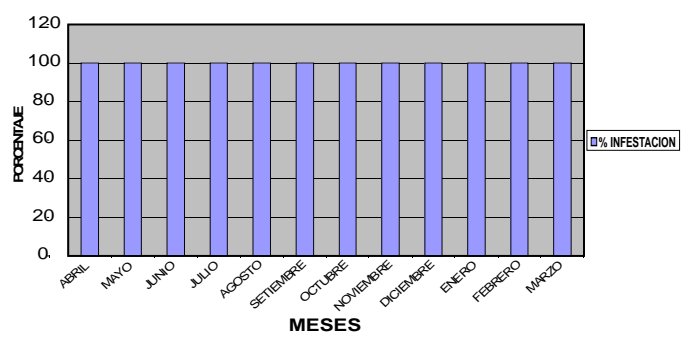

Grafico 1. Infestación de frutos de guanábano en la zona de Satipo.

En el gráfico 2, se puede apreciar que la mosca amarilla del caimito se presenta de noviembre a marzo, época de frutos maduros, donde a medida que pasa el tiempo la infestación aumenta llegando al 100\%; al inicio, cuando las larvas son pequeñas se puede consumir la pulpa, pero cuando éstas desarrollan y alcanzan la semilla, todo el fruto queda destruido por la pudrición secundaria, que impide su comercialización. Esta misma mosca se ha encontrado ocasionalmente en frutos caídos de guanábano, lo que implica que lo toma como hospedero alternante. En muy bajo porcentaje se ha encontrado a la mosca Anastrepha leptozona Hendel.

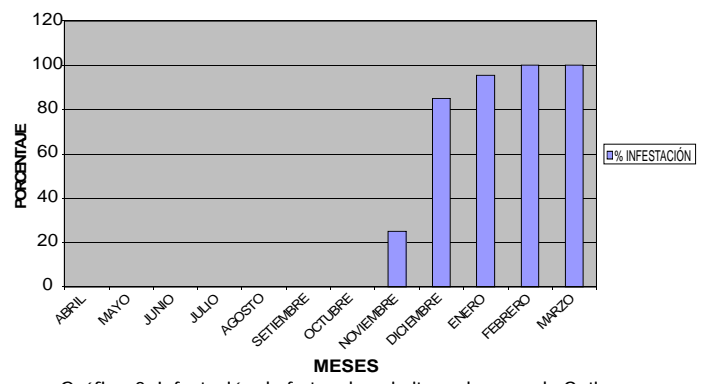

Gráfico 2. Infestación de frutos de caimito en la zona de Satipo

El gráfico 3, nos muestra que el zapote al igual que el caimito tiene frutos maduros sólo en una parte del año, donde la plaga principal es la mosca de la fruta Anastrepha kuhlmanni Lima, cuyo nivel de infestación alcanza el 56\%. Este insecto comparte espacios con la polilla del zapote, que al estado larval barrena los frutos llegando a la semilla.

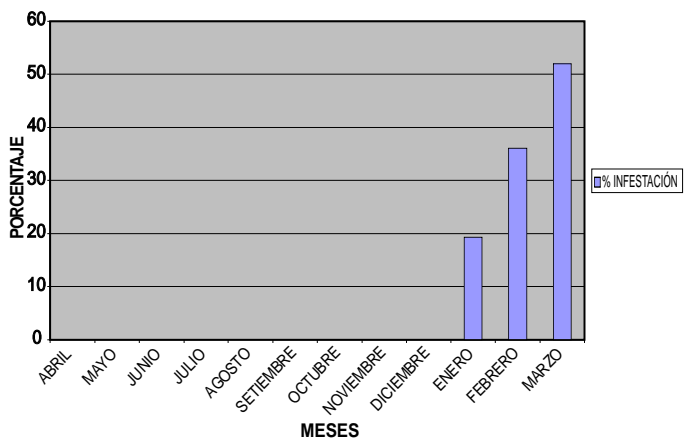

Grafico 3. Infestación de frutos de zapote en la zona de Satipo. 
El gráfico 4, nos muestra la fluctuación poblacional de la polilla del guanábano Cerconata anonella, donde las máximas poblaciones se encuentran desde diciembre a mayo; mientras que las poblaciones mínimas se tienen entre julio y noviembre. Estos datos están en directa relación con la cantidad de frutos en la planta.

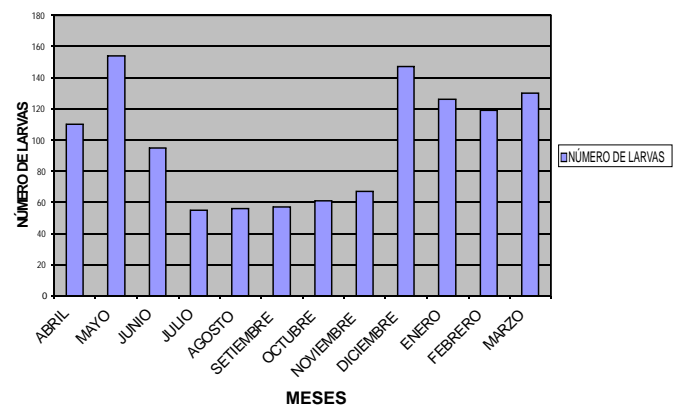

Gráfico 4. Fluctuación poblacional de la polilla del guanábano en la zona de Satipo.

El gráfico 5, nos muestra que la mosca amarilla del caimito alcanza su pico poblacional en los meses de enero y febrero; el resto del año no se encuentra en el caimito, por lo que requiere de hospederos alternantes.

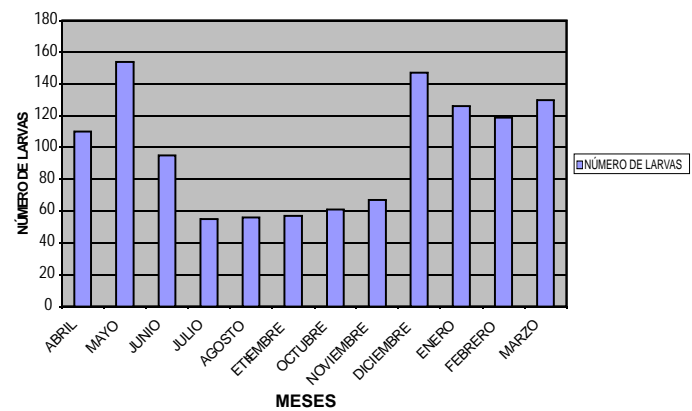

Gráfico 5. Fluctuación poblacional de la mosca amarilla del caimito.

En el gráfico 6, se puede apreciar que la mosca de la fruta en el zapote Anastrepha kuhlmanni Lima se presenta sólo en los tres meses del año, el resto del tiempo debe alojarse en hospederos alternantes aún no determinados. Su pico poblacional lo alcanza en el mes de febrero.

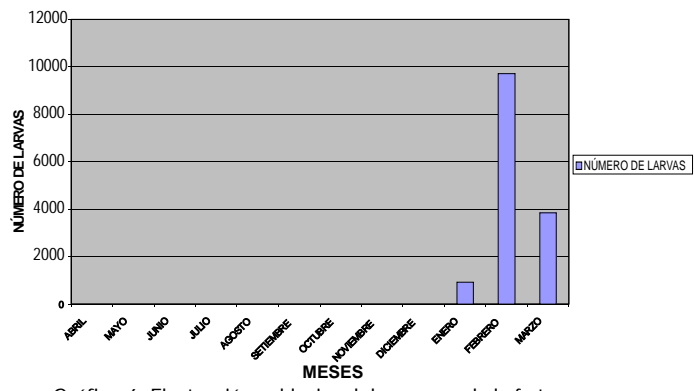

Gráfico 6. Fluctuación poblacional de moscas de la fruta en el zapote en la zona de Satipo.

\section{DISCUSIÓN}

En los frutos de Anona muricata L. el lepidóptero Cerconata anonnella Sepp. fue reportado por varios autores en otros países como Costa Rica (Coto, D. y Saunders J. L, 2001), Ecuador ( PROEXANT, 2000) y en el Perú (Minag, 2001), este último coincide en señalar que se trata de la plaga más importante del guanábano de nuestro país.

El díptero recolectado de frutos caídos no identificado, a nivel larval es blanco cremoso eruciforme, pero más grande que las del género Anastrepha, la morfología no concuerda con las claves taxonómicas de Beingolea G, (1994) y Korytrowski, C (1992). Lo que implica una nueva familia de moscas plagas en frutos.

En frutos de Chrysophillum caimito el díptero registrado es la plaga principal y tiene las mismas características que el registrado en el guanábano, por lo que dada su gran predominancia en el caimito toma como hospedero alternante a frutos caídos de guanábano. La mosca del caimito a diferencia de Anastrepha spp. barrena las semillas del fruto y completa su desarrollo dentro de éstas. Esta información no concuerda con otros autores que manifiestan que daños similares lo realiza un gorgojo picudo Coleoptera - Curculionidae. Este insecto también no fue reportado en la zona de Satipo por Pariona, V. (2004). Sin embargo, fue hallado junto con esta plaga Anastrepha leptozona Hendel, aunque en menor proporción; esta especie coincide con lo hallado con Pariona, V (2004) en la zona, pero no con lo reportado en Venezuela por Caraballo (2004), donde se cita a Anastrepha serpentina Wiedeman.

En el zapote Matisia cordata la mosca Anastrepha kuhlmanni Lima se presenta con mayor frecuencia, con una gran cantidad de larvas por fruto, este dato coincide con Pariona, V. (2004). El lepidóptero registrado tiene características morfológicas parecidas a la polilla del guanábano, pero la larva barrena la cáscara, pulpa y semilla del zapote, donde completa su desarrollo. En la cáscara deja un orificio visible a diferencia de la mosca de la fruta. No hay autores que citan a esta plaga.

La infestación de frutos de guanábano por la polilla del fruto es al $100 \%$, dado que todos los frutos colectados presentaron daños y larvas, esto coincide con lo mencionado por Boscan de Martínez, N. y Godoy J. F (1983) en Venezuela; esto debido a que el guanábano es una planta que presenta frutos todo el año, lo que permite que la polilla haga varios ciclos biológicos al año. Aunque los daños son parciales en la pulpa.

La infestación de frutos de caimito se inicia en el mes de noviembre con $25 \%$ al presentarse los primeros frutos y sube hasta $100 \%$ en el mes de marzo, cuyos daños se 
calculan en $37,02 \%$, lo que evidencia las grandes pérdidas que ocasiona la mosca a este frutal nativo, estos datos son similares con los manifestado por Carballo V. M. y Coto A. D. (1987).

Los frutos infestados de zapote inician con la maduración de los primeros frutos en el mes de enero con $19 \%$, alcanzando un $52 \%$ en el mes de marzo, lo que evidencia las grandes pérdidas de este frutal y los problemas de calidad en la comercialización. Al respecto no hay autores aún que hallan investigado sobre plagas del zapote.

El número de larvas de la polilla Cerconata anonnella Sepp. fluctúa en función a la cantidad de frutos en la zona, siendo el guanábano un frutal que produce todo el año, aunque disminuye entre julio y noviembre, la polilla encuentra alimento disponible todo el año. Al respecto no hay investigaciones sobre fluctuación poblacional de esta plaga. La población de moscas del caimito surge con la aparición de los primeros frutos en diciembre, alcanzando su pico máximo en enero para descender en febrero, estos datos no coinciden con lo manifestado por Pariona V. (2004), quien manifiesta que el pico de maduración del caimito se da en el mes de marzo. Las larvas registradas con mayor frecuencia son de la mosca amarilla y no las de Anastrepha leptozona Hendel que se registraron en menor proporción.

La mosca del zapote Anastrepha kuhlmanni Lima aparece en enero con los primeros frutos de zapote alcanzando su pico máximo en febrero para luego descender en marzo, esto concuerda con los datos de Pariona V. (2004). Al igual que las plagas del caimito, la población de moscas está en directa relación a la cantidad de frutos maduros en la planta. Asimismo, la polilla del zapote se ha encontrado en un $22 \%$ de frutos compartiendo simultáneamente a veces el mismo fruto con la mosca que se alimenta de la pulpa y las larvas de la polilla de la semilla.

\section{LITERATURA CITADA}

Beingolea, G., O. 1994. Guía práctica para identificar insectos de interés agrícola. Lima: RAAA;

Boscán de Martínez, N. y Godoy J. F. 1983. Enemigos naturales de Cerconata anonella Sepp. Perforador del fruto de la guanábano. FONAIAP. Centro Nacional de Investigaciones Agropecuarias. Macaray Venezuela. Disponible en: www.red.pavpolar.info.velagrotrop/v33.

Caraballo, J. Bioline. 2001. Entomotropica Vol 16, № 3 , Sociedad Venezolana de Entomología. Disponible en: www.bioline.org.br/request?em01028.
Carballo, V. M. Y Coto, A. D. 1997 Tolerancia de germoplasma de sapotáceas a Conotraechelus y otros insectos. Disponible en: www.catie.ac.cr/informes/RMIP/ncarba-2-thm.

Cisneros, V., Fausto, H. 1995. Control de plagas agrícolas. Lima: 2da. Edición

Coto, D. y Saunders, J. L., 2001. Insectos plagas de la guanábano en Costa Rica. Revista de Manejo Integrado de Plagas $\mathrm{N}^{0}$ 61. Disponible en: www.catie.ac.cr/inforemación/RMIP.

Korytrowski, C. 1992 Clave para la identificación de especies del género Anastrepha schiner 1968", Panamá.

Mag. 1991 Aspectos técnicos sobre cuarentena y cinco cultivos agrícolas de Costa Rica. Dirección general de investigación y extensión agrícola. Ministerio de agricultura y ganadería. San José de Costa Rica. Disponible en: www.mag.go.cr/bibliotecavirtual.ciencia.tecnologia-guanabana.

Minag. 2001. Cultivo de guanábano. Recursos Naturales. Ministerio de Agricultura de la República del Perú. Disponible en: www.minag.gob.pe/rmn-guanabanasthml.

Pariona, V. R. 2004 Determinación de moscas de la fruta del género Anastrepha y sus hospederos en el valle de Satipo. Tesis para optar el Título profesional de Ingeniero Agrónomo, Facultad de Ciencias Agrarias Universidad Nacional del Centro del Perú, Satipo Perú,

Proexant. Corporación, Guanábano. 2000. Producción de exportaciones agrícolas no tradicionales. Disponible en:

www.proexant.org.ec/manual\%20de\%20guanaban a html-140k.

Sibaja, Ch., G. y Sanabria, U., C. 2002 Principales plagas de insectos, ácaros y moluscos de cultivos agrícolas y forestales de Costa Rica. Servicio Fitosanitario del Estado. San José de Costa Rica. Disponible en: www.protecnet.go.cr/plaga/listado.entomog. 